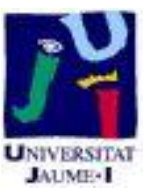

Título artículo / Títol article: Comparison theory of Lorentzian distance with applications to spacelike hypersurfaces

Autores / Autors

Luis J. Alías, Ana Hurtado, Vicente Palmer

Revista:

AIP conference proceedings

Versión / Versió:

Post-print del autor

Cita bibliográfica / Cita bibliogràfica (ISO 690):
ALÍAS, Luis J.; HURTADO, Ana; PALMER, Vicente. Comparison theory of Lorentzian distance with applications to spacelike hypersurfaces. AIP conference proceedings, 2009

url Repositori UJI: http://hdl.handle.net/10234/70280 


\title{
Comparison theory of Lorentzian distance with applications to spacelike hypersurfaces
}

\author{
Luis J. Alías*, Ana Hurtado ${ }^{\dagger}$ and Vicente Palmer** \\ *Departamento de Matemáticas, Universidad de Murcia, E-30100 Espinardo, Murcia, Spain. \\ ljalias@um.es \\ †Departamento de Geometría y Topología, Universidad de Granada, E-18071 Granada, Spain. \\ ahurtado@ugr.es \\ **Departament de Matemàtiques, Universitat Jaume I, E-12071 Castelló, Spain. \\ palmer@mat.uji.es
}

\begin{abstract}
In this paper we summarize some comparison results for the Lorentzian distance function in spacetimes, with applications to the study of the geometric analysis of the Lorentzian distance on spacelike hypersurfaces. In particular, we will consider spacelike hypersufaces whose image under the immersion is bounded in the ambient spacetime and derive sharp estimates for the mean curvature of such hypersurfaces under appropriate hypotheses on the curvature of the ambient spacetime. The results in this paper are part of our recent work [1], where complete details and further related results may be found.
\end{abstract}

Keywords: Lorenzian distance function, Hessian and Laplacian comparison results, spacelike hypersurface, mean curvature, Omori-Yau maximum principle.

PACS: $04.20 . \mathrm{Cv}, 02.40 . \mathrm{Vh}$

\section{THE LORENTZIAN DISTANCE FUNCTION}

Consider $M^{n+1}$ an $(n+1)$-dimensional spacetime, and let $p, q$ be points in $M$. Using the standard terminology and notation from Lorentzian geometry, one says that $q$ is in the chronological future of $p$, written $p \ll q$, if there exists a future-directed timelike curve from $p$ to $q$. Similarly, $q$ is in the causal future of $p$, written $p<q$, if there exists a future-directed causal (i.e., nonspacelike) curve from $p$ to $q$. Obviously, $p \ll q$ implies $p<q$. As usual, $p \leq q$ means that either $p<q$ or $p=q$.

For a subset $S \subset M$, one defines the chronological future of $S$ as $I^{+}(S)=\{q \in M: p \ll$ $q$ for some $p \in S\}$, and the causal future of $S$ as $J^{+}(S)=\{q \in M: p \leq q$ for some $p \in S\}$. Thus $S \cup I^{+}(S) \subset J^{+}(S)$. In particular, the chronological future $I^{+}(p)$ and the causal future $J^{+}(p)$ of a point $p \in M$ are

$$
I^{+}(p)=\{q \in M: p \ll q\}, \quad \text { and } \quad J^{+}(p)=\{q \in M: p \leq q\} .
$$

As is well-known, $I^{+}(p)$ is always open, while $J^{+}(p)$ is neither open nor closed in general.

If $q \in J^{+}(p)$, then the Lorentzian distance $d(p, q)$ is the supremum of the Lorentzian lengths of all the future-directed causal curves from $p$ to $q$ (possibly, $d(p, q)=+\infty$ ). If $q \notin J^{+}(p)$, then the Lorentzian distance $d(p, q)=0$ by definition. In particular, $d(p, q)>0$ if and only if $q \in I^{+}(p)$. Let us recall that the Lorentzian distance function $d: M \times M \rightarrow[0,+\infty]$ for an arbitrary spacetime may fail to be continuous in general, 
restrict this function on certain special subsets of $M$. Let $\eta$ be the future-directed Gauss map of $S$, and let $s: S \rightarrow[0,+\infty]$ be the function given by

$$
s(p)=\sup \left\{t \geq 0: d_{S}\left(\gamma_{p}(t)\right)=t\right\},
$$

where $\gamma_{p}:[0, a) \rightarrow M$ is the future inextendible geodesic starting at $p$ with initial velocity $\eta_{p}$. Then, one can define

$$
\tilde{\mathscr{I}}^{+}(S)=\left\{t \eta_{p}: \text { for all } p \in S \text { and } 0<t<s(p)\right\}
$$

and consider the subset $\mathscr{I}^{+}(S)=\exp _{S}\left(\operatorname{int}\left(\tilde{I}^{+}(S)\right)\right) \subset I^{+}(S)$, where $\exp _{S}$ denotes the exponential map with respect to the hypersurface $S$. Below we collect some interesting properties about $d_{S}$ (see [4, Section 3.2]).

Lemma 11 Let $S$ be an achronal spacelike hypersurface in a spacetime $M$.

1. If $S$ is compact and $M$ is globally hyperbolic, then $s(p)>0$ for all $p \in S$ and $\mathscr{I}^{+}(S) \neq \emptyset$.

2. If $\mathscr{I}^{+}(S) \neq \emptyset$, then $d_{S}$ is smooth on $\mathscr{I}^{+}(S)$ and its gradient $\bar{\nabla} d_{S}$ is a past-directed timelike (geodesic) unit vector field on $\mathscr{I}^{+}(S)$.

Doing now a similar analysis of the Lorentzian distance function to an achronal hypersurface, one can derive also sharp estimates for the mean curvature of spacelike hypersurfaces which contained in its chronological future. Further details about this may be found in [1].

\section{ACKNOWLEDGMENTS}

This research is a result of the activity developed within the framework of the Programme in Support of Excellence Groups of the Región de Murcia, Spain, by Fundación Séneca, Regional Agency for Science and Technology (Regional Plan for Science and Technology 2007-2010). L.J. Alías was partially supported by MEC-DGI project MTM2007-64504, and Fundación Séneca project 04540/GERM/06, Spain. A. Hurtado and V. Palmer were partially supported by Spanish MEC-DGI project MTM2007-62344, and the Bancaixa-Caixa Castelló Foundation, Spain.

\section{REFERENCES}

1. L.J. Alías, A. Hurtado, and V. Palmer, Trans. Amer. Math. Soc. in press. Available at http://arxiv.org/pdf/0802.4376.

2. B. O'Neill, Semi-Riemannian Geometry; With Applications to Relativity, Academic Press, New York, 1983.

3. J.K. Beem, P.E. Ehrlich, and K.L. Easey, Global Lorentzian Geometry, Marcel Dekker Inc., New York, 1996.

4. F. Erkekoglu, E. García-Río, and D. N. Kupeli, Gen. Relativ. Gravit. 35, 1597-1615 (2003).

5. S. Pigola, M. Rigoli, and A.G. Setti, Memoirs Amer. Math. Soc. 822 (2005).

6. H. Omori, J. Math. Soc. Japan 19, 205-214 (1967).

7. S.T. Yau, Commun. Pure Appl. Math. 28, 201-228 (1975).

8. J. A. Aledo, and L. J. Alías, Manuscripta Math. 101, 401-413 (2000). 